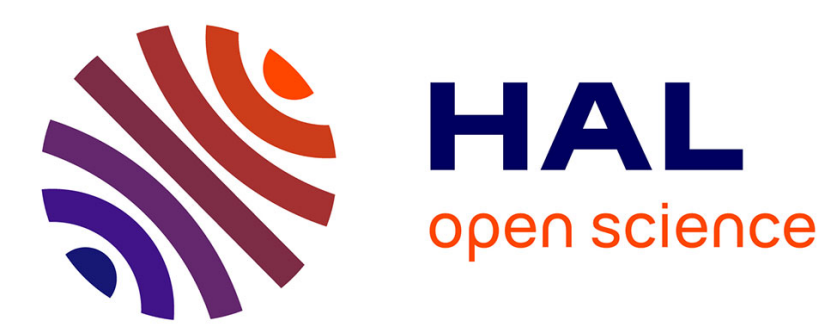

\title{
Analysis of Small-World Features in Vehicular Social Networks
}

\author{
Anna Maria Vegni, Valeria Loscrì, Pietro Manzoni
}

\section{To cite this version:}

Anna Maria Vegni, Valeria Loscrì, Pietro Manzoni. Analysis of Small-World Features in Vehicular Social Networks. CCNC 2019 - IEEE Consumer Communications \& Networking Conference, Jan 2019, Las Vegas, United States. hal-01907422

\section{HAL Id: hal-01907422 \\ https://hal.science/hal-01907422}

Submitted on 22 Nov 2018

HAL is a multi-disciplinary open access archive for the deposit and dissemination of scientific research documents, whether they are published or not. The documents may come from teaching and research institutions in France or abroad, or from public or private research centers.
L'archive ouverte pluridisciplinaire HAL, est destinée au dépôt et à la diffusion de documents scientifiques de niveau recherche, publiés ou non, émanant des établissements d'enseignement et de recherche français ou étrangers, des laboratoires publics ou privés. 


\title{
Analysis of Small-World Features in Vehicular Social Networks
}

\author{
Anna Maria Vegni*, Valeria Loscrí ${ }^{\dagger}$, and Pietro Manzoni ${ }^{\ddagger}$ \\ *Department of Engineering, COMLAB Telecommunications Lab \\ Roma Tre University, Rome, Italy. Email: annamaria.vegni@uniroma3.it \\ ${ }^{\dagger}$ INRIA Lille-Nord Europe, FUN Research Lab, Lille Nord-Europe, France. Email: valeria.loscri@inria.fr \\ ‡Universitat Politécnica de Valéncia, Computer Engineering Department, Valencia, Spain. Email: pmanzoni@disca.upv.es
}

\begin{abstract}
The Internet-of-Vehicles (IoV) allows vehicles to share information for common services, such as traffic status or safety alerts, not only among themselves but also with neighboring devices and users. Vehicular Social Networks (VSNs) appear when IoVs is combined with social features like chat, sharing achievements, wish-listings, etc. In this work, we demonstrate that VSNs require specific data dissemination techniques. To this aim, we consider the Barabási model and we study the impact of its use for data dissemination in VSNs. Moreover, the use of a probabilistic data dissemination protocol based on social features [1] proves that the time-evolving dynamics of the vehicular social network graph presents a small-world structure, where nodes tend to connect through clusters whose average distance is low on average. This result highlights how VSNs are a specific class of vehicular ad hoc networks with peculiar features that distinguish them from classical online social networks.
\end{abstract}

\section{INTRODUCTION}

Social networks offer the opportunity to distribute information, direct traffic, create a dialogue with specific groups, and deploy applications that allow users to participate directly in a coordinated way. However, when we change the context, and pass from online social networks (OSNs) [2] to mobile/vehicular social networks (VSNs) [3], the quest for an efficient and effective specific solution to distribute information is clearly a crucial issue.

In this paper, we analyze SCARF from a network graph evolution perspective and observe that the obtained time-evolving VSN follows a small-world behavior [4]. According to Watts and Strogatz [5], small-world networks are a class of networks that are "highly clustered, yet have small characteristic path lengths, like random graphs". These characteristics result in networks with unique properties of regional specialization with efficient information transfer. Determining whether a network is a "small-world" is important in understanding the system's behavior, and can provide useful data, like the diameter, or path length, of a network. Moreover, knowing the network's general topology allows to better understand the overall characteristics and behavior of such systems. In our context, spotting a congestion in specific links allows to forecasts possible critical situations if those links fail and prepare the network for failure tolerance.

This paper is organized as follows. Section II describes how the small-world model can be applied into VSNs. We start from the Barabási-Albert model, which generates scalefree networks based on the growth and preferential attachment features, and we inquire if this model can also be suitable for VSNs. Through an analytical analysis, in Section III we demonstrate that the time-evolving graph theoretic model with SCARF carries to a small-world structure, then stating SCARF is more suitable for VSNs, instead of the Barabási-Albert model. Finally, conclusions are drawn at the end of the paper.

\section{SMALL-WORLD MODEL FOR VSNS}

Let a VSN be modeled through the Barabási-Albert (BA) model, thus generating a scale-free vehicular social network. The VSN is initially made of $m_{0}$ vehicles. The BA model states that a vehicle attempting to connect will be likely linked to existing vehicles already connected to the VSN.

The probability that the $l$-th link (with $l=1,2, \ldots, m_{0}$ ) of the new vehicle connects to the $i$-th vehicle in the VSN depends on the degree $k_{i}$ as [4]:

$$
\prod\left(k_{i}\right)=\frac{k_{i}}{\sum_{j} k_{j}}, \quad \text { with } j \neq i .
$$

Eq. (1) can be translated into the preferential attachment feature of the BA model, so that a new node tends to link to the "most" connected nodes (i.e., the nodes with higher degree). In this way, there is a node evolution towards hubs. Hubs represent the nodes with highest degree, and are able to attract new nodes accessing the network. This reflects the richgets-richer phenomenon, so that new nodes are more likely to connect to the "most" connected nodes than to the "least" connected nodes.

After $t$ time steps, the BA model generates a network with $N=t+m_{0}$ nodes and $\left(m_{0}+m t\right)$ links. Considering the time evolution of the VSN, the probability that a node $v$ will be connected to the $i$-th node will be:

$$
p_{v i}=\frac{k_{i}}{2 t-1} \text {, with } v \neq i
$$

which represents the linearized chord diagram (LCD) [6], where we did not consider the existence of self-loops.

In this context, we can apply SCARF as a message rebroadcasting technique towards selected vehicles showing a social degree that makes them "social vehicles". SCARF 
distinguishes different types of vehicles, based on their social features, such as relationships (i.e., a friend vehicle or a family vehicle), common interests (i.e., a classmate vehicle or a commuter vehicle) and social activity (i.e., a hub vehicle) [1].

\section{ANALYTICAL PERFORMANCE}

In this section, we present the analytical results obtained in a vehicular social network, following the time-evolving BA model. Our aim is to demonstrate that SCARF approach is well-suitable for data dissemination in VSNs, as it takes into account not only vehicular features, but also social parameters, and thus it allows the VSN to evolve in a small-world structure. Compared to typical approaches for small-world networks, SCARF shows that the network evolution follows a smallworld nature, i.e., the distance between two randomly chosen nodes in the network is short, and the nodes tend to form more clusters.

Let us consider a network graph comprised of 50 nodes. Then, five new nodes will enter the VSN, trying to connect to existing nodes. In this scenario, we compare SCARF technique to the BA model. Analytical results are expressed in terms of average ( $i$ ) distance, and (ii) clustering coefficient, for different network sizes (i.e., from 50 to 55 nodes). These metrics were chosen in order to assess how many vehicles are connected, how their links evolve, how central and important a node is. This analysis aims to determine if SCARF produces a VSN with a small-world structure that is when nodes have a higher degree, form clusters, and present a lower distance on average, as stated by the following definition.

Definition 1 (Small-world structure): A network graph has a small-world structure if nodes tend forming clusters and the average distance is low. Similarly, a network graph with nodes having low betweenness and high centrality presents a small-world structure.

In SCARF, each vehicle entering the VSN tries to connect to high social degree nodes, which also have high network degree. It follows that the average distance with SCARF for different vehicles entering the VSN is the lowest with respect to the BA approach. In Fig. 1, the BA model shows highest average distance. This means that SCARF highlights a smallworld behavior in VSNs, so that nodes are most connected to each others. Also, considering the average diameter $D$ (i.e., the maximum average distance) in the network with 55 nodes, we obtain $D_{S}=42.20$ and $D_{B}=44.23$, using SCARF and BA techniques, respectively.

The clustering coefficient captures the degree to which the neighbors of a given node link to each other. The local clustering coefficient gives information if two neighbors of a node link to each other, and it is one of the most important metrics for the social network analysis. Fig. 2 depicts the average clustering coefficient for different network scenarios. This is a measure of the degree to which nodes tend to cluster together. Highest performance is obtained with SCARF approach, which provides a clustering coefficient of $\approx 0.8$, value that keeps increasing for higher network sizes, and

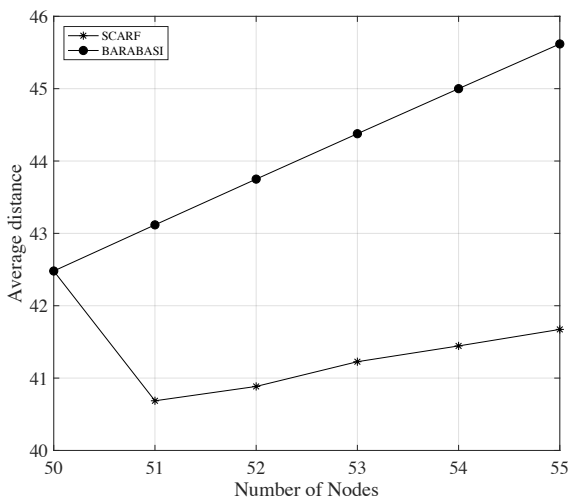

Fig. 1: Average distance in different network scenarios.

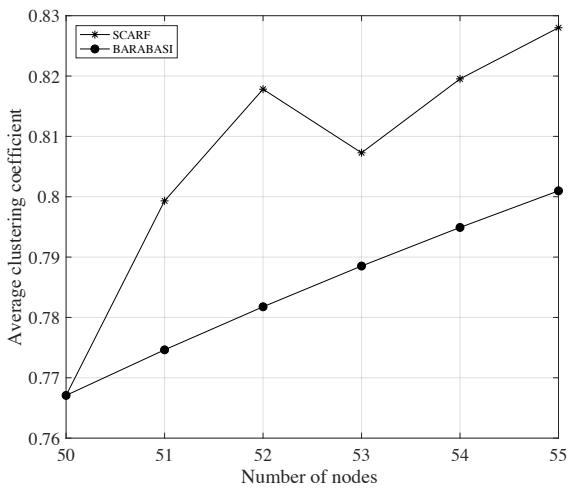

Fig. 2: Average clustering coefficient in different network scenarios.

represents that the neighbors of the $i$-th node link to each other. In contrast, lower values are experienced for the BA model, meaning that neighboring vehicles limit the clustering and to connect to each others.

\section{CONCLUSIONS}

In this paper, we focused on the key role played by an effective dissemination scheme in a VSN context.

We first analyzed SCARF from a graph evolution perspective and we observed that the obtained time-evolving VSN follows a small-world behavior [4]. Then, we compared the performance of SCARF to the BA model. Best performance is with the SCARF technique, since the time-evolving dynamics of the vehicular social network graph allow forming a smallworld structure, where nodes tend to connect through clusters and the average distance is lower.

\section{REFERENCES}

[1] A. M. Vegni, V. Loscrí, and R. Petrolo, "SCARF: A SoCial-Aware Reliable Forwarding Technique for Vehicular Communications," in Proc. of the 3rd Workshop on Experiences with the Design and Implementation of Smart Objects. ACM, 2017, pp. 1-6.

[2] S. Staab et al., "Social networks applied," IEEE Intell. Syst., vol. 20, no. 1, pp. 80-93, 2005.

[3] A. M. Vegni and V. Loscrí, "A survey on vehicular social networks," IEEE Communications Surveys Tutorials, vol. 17, no. 4, pp. 2397-2419, Fourthquarter 2015. 
[4] A.-L. Barabási, Network Science. Cambridge University Press, 2016.

[5] D. J. Watts and S. H. Strogatz, "Collective dynamics of "small-world" networks," Nature, vol. 393, no. 6684, pp. 440-442, June 1998.
[6] B. Bollobás, O. Riordan, J. Spencer, and G. Tusnády, "The degree sequence of a scale-free random graph process," Random Structures and Algorithms, vol. 18, pp. 279-290, 2001. 